\title{
Estimation of the Inverse Weibull Parameters Under Ranked Set Sampling
}

\author{
Samia A. Salem ${ }^{1}$, Mazen Nassar ${ }^{1}$ and Hanem E. Hozaien ${ }^{2}$ \\ ${ }^{1}$ Department of Statistics, Faculty of Commerce, Zagazig University, Egypt. \\ ${ }^{2}$ Department of Statistics, Faculty of Commerce, Al-Azhar University, Egypt.
}

\begin{abstract}
In this paper we use the maximum likelihood (ML) and the modified maximum likelihood (MML) methods to estimate the unknown parameters of the inverse Weibull (IW) distribution as well as the corresponding approximate confidence intervals. The estimates of the unknown parameters are obtained based on two sampling schemes, namely, simple random sampling (SRS) and ranked set sampling (RSS). Comparison between the different proposed estimators is made through simulation via their mean square errors (MSE), Pitman nearness probability (PN) and confidence length.
\end{abstract}

Keywords: Inverse Weibull, maximum likelihood, modified maximum likelihood, ranked set sampling. 


\section{Introduction}

Ranked set sampling is recognized as a useful sampling technique for improving the precision and increasing the efficiency of estimation when the variable under consideration is expensive to measure or difficult to obtain but cheap and easy to rank. Ranked set sampling has been suggested by McIntyre (1952) in relation to estimating pasture yields. Takahasi and Wakimoto (1968) established the theory of RSS, they showed that the sample mean of RSS is an unbiased estimator for the population mean and is more efficient than the sample mean of SRS. According to RSS, we first select $m^{2}$ elements denoted by $x_{i(i)},(i=1,2, \ldots, m)$ from the population at random. These elements are then randomly splitted into $m$ sets of $m$ units each. On each set, we rank the $m$ units by judgment or a supporting variable according to the characteristic of interest. We select the element with the smallest ranking, $x_{1(1)}$, for measurement from the first set. From the second set we select the element with the second smallest ranking, $x_{2(2)}$. We continue in this way until we have ranked the elements in the $m$ th set and selected the element with the largest ranking, $x_{m(m)}$, as in Figure 1. This complete procedure, called a cycle which is repeated independently $k$ times to obtain a ranked set sample of size $n=m k$ (see Chen et al. (2004)).

\begin{tabular}{c}
\hline One cycle \\
\hline$x_{1(1)}$ \\
$x_{2(2)}$ \\
$\vdots$ \\
$x_{m(m)}$ \\
\hline
\end{tabular}

Figure 1: Ranked set sampling

Marginally $x_{i(i)}(i$ th order statistics of the $i$ th random sample in a sample of size $m$ ) have the same distribution with pdf given by (see David and Nagaraja (2003)).

$$
f_{m}\left(x_{i(i)} ; \theta\right)=\frac{m !}{(i-1) !(m-i) !}\left[F\left(x_{i(i)} ; \theta\right)\right]^{i-1}\left[1-F\left(x_{i(i)} ; \theta\right)\right]^{m-i} f\left(x_{i(i)} ; \theta\right),-\infty<x<\infty
$$

Many authors have studied the RSS and its modifications; for example, Al-Saleh and Al-Hadrami (2003a) studied the ML and MML estimation of location parameters of symmetric distribution using moving extremes ranked set sampling and SRS. Al-Saleh et al. (2003b) studied ML and MML estimation of the mean of exponential distribution based on moving extremes ranked set sampling under both perfect and imperfect ranking. Helu et al. (2010) studied estimation of the parameters of Weibull distribution using different methods of estimation based on SRS, RSS and modified RSS. Abu-Dayyeh and Assrhani (2013) considered estimation of the shape and location parameters of the Pareto distribution based on SRS and RSS. Balci et al. (2013) used RSS, ranked set sampling by choosing both diagonal elements and ranked set sampling by choosing extremes of the samples to derive the MML estimates (MMLEs) for the population mean and variance of normal distribution. Hussian (2014) discussed Kumaraswamy distribution using SRS and RSS techniques based on maximum likelihood and Bayesian estimation methods. The moving extremes ranked set 
sampling was presented by Chen et al. (2014) to obtain the MMLEs for some usual scale distributions. Sadek and Alharbi (2014) studied the problem of Bayesian estimation of the parameters for Weibull distribution based on RSS under squared error loss function and LINEX loss function. Dey et al. (2016) considered RSS, modified ranked set sampling, median ranked set sampling and SRS to estimate the parameter of Rayleigh distribution.

The inverse Weibull distribution (it is also known as Fre'chet distribution) is more appropriate than the Weibull distribution for modeling a non-monotone and unimodal hazard rate functions. The probability density function (pdf) and cumulative distribution function (cdf) of the IW distribution are, respectively, given by

$$
f(x)=\lambda \theta x^{-(\theta+1)} e^{-\lambda x^{-\theta}}, x>0, \lambda, \theta>0,
$$

and

$$
F(x)=e^{-\lambda x^{-\theta}}
$$

where $\lambda$ is the scale parameter and $\theta$ is the shape parameter. Such model has been suggested as a model in the analysis of life testing data. Keller and Kamath (1982) introduced the IW distribution for modelling reliability data and failures of mechanical components subject to degradation. Estimation of the parameters of IW distribution in classical and Bayesian methods has been discussed in literature. Calabria and Pulcini (1989) discussed the statistical properties of the MLEs of the parameters and reliability for a complete sample. Erto (1989) used the Least Square method to obtain the estimators of the parameters and reliability. Calabria and Pulcini (1990) obtained the MLEs and the Least Square of the parameters. Calabria and Pulcini (1992) derived the Bayes estimators of the parameters and reliability. The hazard rate function of the IW distribution is

$$
h(x)=\lambda \theta x^{-(\theta+1)}\left(e^{\lambda x^{-\theta}}-1\right)^{-1} .
$$

The shape of the hazard rate function of the IW distribution can be decreasing, increasing or unimodal based on the value of the shape parameter. Many works have been suggested to estimate the unknown parameters of the IW distribution, see for example, Calabria and Pulcini (1994), Maswadah (2003), Singh et al. (2013), Musleh and Helu (2014) and Nassar and AboKasem (2017). For more details see Hassan and Naesr (2013).

The main objective of this study is to obtain the MLEs and MMLEs as well as the approximate confidence intervals for the scale and the shape parameters of the IW distribution based on SRS and RSS. We compare the performance of the different estimators by using a simulation study. The rest of the paper is organized as follows: In Section 2, the MLEs are obtained based on the SRS and RSS. The MMLEs under the SRS and RSS are derived in Section 3. In Section 4, a simulation study is conducted to evaluate the performance of the different estimators. A real data set is analyzed in Section 5 for illustrative purposes. Finally, the conclusion in Section 6.

\section{Maximum likelihood estimation for IW distribution parameters}

In this section, the ML estimation method is used to estimate the IW distribution parameters using SRS and RSS. 


\subsection{Maximum likelihood estimation under SRS}

Let $x_{1}, x_{2}, \ldots, x_{n}$ be a simple random sampling of size $n$ from the IW distribution, then from the pdf in (1) the log likelihood function is

$$
\ln L(\lambda, \theta)=n \ln \lambda+n \ln \theta-(\theta+1) \sum_{i=1}^{n} \ln x_{i}-\lambda \sum_{i=1}^{n} x_{i}^{-\theta} .
$$

The maximum likelihood estimators of $\lambda$ and $\theta$ can be obtained by differentiate (3) with respect to $\lambda$ and $\theta$ and equating the results to zero as follows

$$
\frac{n}{\hat{\lambda}}-\sum_{i=1}^{n} x_{i}^{-\widehat{\theta}}=0
$$

and

$$
\frac{n}{\hat{\theta}}-\sum_{i=1}^{n} \ln x_{i}+\hat{\lambda} \sum_{i=1}^{n} x_{i}^{-\widehat{\theta}} \ln x_{i}=0 .
$$

From equation (4), we can obtain the MLE of $\lambda$ as

$$
\hat{\lambda}=\frac{n}{\sum_{i=1}^{n} x_{i}^{-\widehat{\theta}}} \text {. }
$$

Substituting the value of $\hat{\lambda}$ given by (6) in (5), we can obtain the MLE of the parameter $\theta$.

Now we use the large sample approximation to construct the approximate confidence intervals for the parameters $\lambda$ and $\theta$. The approximate inverse of observed information matrix of the unknown parameters is given by

$$
I^{-1}(\lambda, \theta)=\left(\begin{array}{ll}
-\frac{\partial^{2} \ln L}{\partial \lambda^{2}} & -\frac{\partial^{2} \ln L}{\partial \lambda \partial \theta} \\
-\frac{\partial^{2} \ln L}{\partial \theta \partial \lambda} & -\frac{\partial^{2} \ln L}{\partial \theta^{2}}
\end{array}\right)_{(\lambda, \theta)=(\widehat{\lambda}, \widehat{\theta})}^{-1}
$$

where

and

$$
\begin{gathered}
-\left.\frac{\partial^{2} \ln L}{\partial \lambda^{2}}\right|_{\widehat{\lambda}, \widehat{\theta}}=\frac{n}{\hat{\lambda}^{2}} \\
-\left.\frac{\partial^{2} \ln L}{\partial \theta^{2}}\right|_{\widehat{\lambda}, \widehat{\theta}}=\frac{n}{\hat{\theta}}+\hat{\lambda} \sum_{i=1}^{n} x_{i}^{-\widehat{\theta}}\left(\ln x_{i}\right)^{2}
\end{gathered}
$$

$$
-\left.\frac{\partial^{2} \ln L}{\partial \theta \partial \lambda}\right|_{\widehat{\lambda}, \widehat{\theta}}=-\sum_{i=1}^{n} x_{i}^{-\widehat{\theta}} \ln x_{i}
$$

Thus, the approximate confidence intervals of $\lambda$ and $\theta$, are respectively, given by

$$
\hat{\lambda} \pm z_{v / 2} \sqrt{\operatorname{var}(\hat{\lambda})} \text { and } \hat{\theta} \pm z_{v / 2} \sqrt{\operatorname{var}(\hat{\theta})}
$$


where $\operatorname{var}(\hat{\lambda})$ and $\operatorname{var}(\hat{\theta})$, respectively, are the main diagonal elements in (7) and $z_{v / 2}$ is the upper $v / 2$ percentile of a standard normal distribution.

\subsection{Maximum likelihood estimation under RSS}

Let $x_{1(1)}, x_{2(2)}, \ldots, x_{n(n)}$ be a RSS of size $n$ from the IW distribution with pdf (1) and cdf (2), then the likelihood function ignoring the constant term can be written as

$$
L(\lambda, \theta) \propto \lambda^{n} \theta^{n} e^{-\lambda \sum_{i=1}^{n} x_{i(i)}^{-\theta}} \prod_{i=1}^{n} x_{i(i)}^{-(\theta+1)} \prod_{i=1}^{n}\left[e^{-\lambda x_{i(i)}^{-\theta}}\right]^{i-1} \prod_{i=1}^{n}\left[1-e^{-\lambda x_{i(i)}^{-\theta}}\right]^{n-i}
$$

The log-likelihood function is given by

$$
\begin{aligned}
& \ln L(\lambda, \theta) \propto n \ln (\lambda \theta)-\lambda \sum_{i=1}^{n} i x_{i(i)}^{-\theta}-(\theta+1) \sum_{i=1}^{n} \ln x_{i(i)} \\
& +\sum_{i=1}^{n}(n-i) \ln \left(1-e^{-\lambda x_{i(i)}^{-\theta}}\right)
\end{aligned}
$$

From (9), the likelihood equations can be written as follows

$$
\frac{n}{\hat{\lambda}}-\sum_{i=1}^{n} i x_{i(i)}^{-\widehat{\theta}}+\sum_{i=1}^{n}(n-i)\left(\frac{x_{i(i)}^{-\widehat{\theta}} e^{-\widehat{\lambda} x_{i(i)}^{-\widehat{\theta}}}}{1-e^{-\widehat{\lambda} x_{i(i)}^{-\widehat{\theta}}}}\right)=0
$$

and

$$
\begin{aligned}
& \frac{n}{\hat{\theta}}+\hat{\lambda} \sum_{i=1}^{n} i x_{i(i)}^{-\widehat{\theta}} \ln x_{i(i)}-\sum_{i=1}^{n} \ln x_{i(i)} \\
- & \sum_{i=1}^{n}(n-i)\left(\frac{\hat{\lambda} x_{i(i)}^{-\widehat{\theta}} \ln x_{i(i)} e^{-\widehat{\lambda} x_{i(i)}^{-\widehat{\theta}}}}{1-e^{-\widehat{\lambda} x_{i(i)}^{-\widehat{\theta}}}}\right)=0 .
\end{aligned}
$$

It is to be noted that the likelihood equations in this case cannot be solved explicitly, so the MLEs of $\lambda$ and $\theta$ can be obtained by using any numerical technique. The elements of the observed information matrix with respect to $\lambda$ and $\theta$ are as follow:

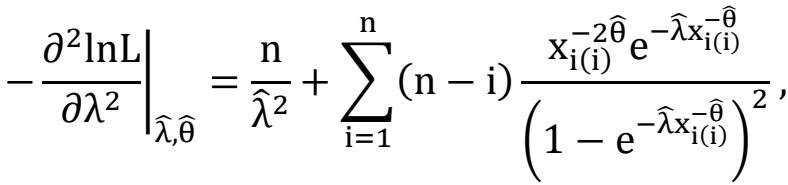

$$
\begin{aligned}
& -\left.\frac{\partial^{2} \ln L}{\partial \theta^{2}}\right|_{\widehat{\lambda}, \widehat{\theta}}=\frac{n}{\hat{\theta}^{2}}+\hat{\lambda} \sum_{i=1}^{n} i x_{i(i)}^{-\widehat{\theta}}\left(\ln x_{i(i)}\right)^{2} \\
& -\hat{\lambda} \sum_{i=1}^{n} \frac{(n-i)\left(\ln x_{i(i)}\right)^{2} x_{i(i)}^{-\widehat{\theta}} e^{-\hat{\lambda} x_{i(i)}^{-\widehat{\theta}}}\left(1-\hat{\lambda} x_{i(i)}^{-\widehat{\theta}}-e^{-\hat{\lambda} x_{i(i)}^{-\widehat{\theta}}}\right)}{\left(1-e^{-\widehat{\lambda} x_{i(i)}^{-\widehat{\widehat{C}}}}\right)^{2}}
\end{aligned}
$$

and 


$$
\begin{aligned}
-\left.\frac{\partial^{2} \ln L}{\partial \lambda \partial \theta}\right|_{\widehat{\lambda}, \widehat{\theta}}= & -\sum_{i=1}^{n} i x_{i(i)}^{-\widehat{\theta}} \ln x_{i(i)} \\
& +\sum_{i=1}^{n} \frac{(n-i) x_{i(i)}^{-\widehat{\theta}} e^{-\widehat{\lambda} x_{i(i)}^{-\widehat{\theta}}} \ln x_{i(i)}\left(1-\hat{\lambda} x_{i(i)}^{-\widehat{\theta}}-e^{-\widehat{\lambda} x_{i(i)}^{-\widehat{\theta}}}\right)}{\left(1-e^{-\widehat{\lambda} x_{i(i)}^{-\widehat{\theta}}}\right)^{2}}
\end{aligned}
$$

\section{Modified maximum likelihood estimation for IW distribution parameters}

In this section, the MMLs of the unknown parameters of the IW distribution are obtained using SRS and RSS.

\subsection{Modified maximum likelihood estimation under SRS}

It is seen that from (5) the ML equation of $\theta$ cannot be obtained in explicit form, therefore, the MMLEs which have explicit form are obtained. Let $Z=-\ln X$, then $Z$ follows the extreme value distribution with pdf and cdf given, respectively, by (see Johnson et al. (1994)).

$$
g(z)=\frac{1}{\sigma} \exp \left\{\left(\frac{z-\mu}{\sigma}\right)-\exp \left(\frac{z-\mu}{\sigma}\right)\right\}, \quad-\infty<z<\infty,
$$

and

$$
G(z)=1-\exp \left\{-\exp \left(\frac{z-\mu}{\sigma}\right)\right\}
$$

where $\mu=-\ln \lambda / \theta$ is the location parameter and $\sigma=1 / \theta$ is the scale parameter. From (12) and under SRS the log-likelihood function is given by

$$
\ln L^{*}(\mu, \sigma)=-n \ln \sigma+\sum_{i=1}^{n} t_{i}-\sum_{i=1}^{n} e^{t_{i}}
$$

where $t_{i}=\left(z_{i}-\mu\right) / \sigma, i=1,2, \ldots, n$. To obtain the MMLEs, we consider $g\left(t_{i}\right)=e^{t_{i}}$, and linearize this function by using Taylor series expansions as

$$
g\left(t_{i}\right)=\alpha_{i}+\beta_{i} t_{i}
$$

where

$$
\alpha_{i}=e^{v_{i}}\left(1-v_{i}\right) \text { and } \beta_{i}=e^{v_{i}} .
$$

Using these linear approximations, the modified likelihood equations can be written as

$$
-\frac{n}{\hat{\sigma}}+\frac{1}{\hat{\sigma}} \sum_{i=1}^{n}\left(\alpha_{i}+\beta_{i} t_{i}\right)=0
$$

and

$$
-\frac{n}{\hat{\sigma}}-\frac{1}{\hat{\sigma}} \sum_{i=1}^{n} t_{i}+\frac{1}{\hat{\sigma}} \sum_{i=1}^{n} t_{i}\left(\alpha_{i}+\beta_{i} t_{i}\right)=0
$$

The solutions of equations (14) and (15) are

and

$$
\hat{\mu}_{M M L-S R S}=\frac{\sum_{i=1}^{n} \beta_{i} z_{i}}{\sum_{i=1}^{n} \beta_{i}}+\hat{\sigma} \frac{\left(\sum_{i=1}^{n} \alpha_{i}-n\right)}{\sum_{i=1}^{n} \beta_{i}}
$$


where

$$
\hat{\sigma}_{M M L-S R S}=\frac{-V+\sqrt{V^{2}+4 n W}}{2 n}
$$

$$
V=\left[\sum_{i=1}^{n}\left(z_{i}-\hat{\mu}\right)+\sum_{i=1}^{n} \alpha_{i}\left(z_{i}-\hat{\mu}\right)\right] \text { and } W=\sum_{i=1}^{n} \beta_{i}\left(z_{i}-\hat{\mu}\right)^{2} .
$$

From the modified likelihood equations (14) and (15), we can obtain the elements of the observed information matrix as follows

and

$$
\begin{gathered}
-\left.\frac{\partial^{2} \ln L^{*}}{\partial \mu^{2}}\right|_{\widehat{\mu}, \widehat{\sigma}} \cong \frac{1}{\hat{\sigma}^{2}} \sum_{i=1}^{n} \beta_{i} \\
-\left.\frac{\partial^{2} \ln L^{*}}{\partial \sigma^{2}}\right|_{\widehat{\mu}, \widehat{\sigma}} \cong-\frac{n}{\hat{\sigma}^{2}}-\frac{2}{\hat{\sigma}^{2}} \sum_{i=1}^{n} t_{i}+\frac{2}{\hat{\sigma}^{2}} \sum_{i=1}^{n} \alpha_{i} t_{i}+\frac{3}{\hat{\sigma}^{2}} \sum_{i=1}^{n} \beta_{i} t_{i}^{2}
\end{gathered}
$$

$$
-\left.\frac{\partial^{2} \ln L^{*}}{\partial \mu \partial \sigma}\right|_{\widehat{\mu}, \widehat{\sigma}} \cong-\frac{n}{\hat{\sigma}^{2}}+\frac{1}{\hat{\sigma}^{2}} \sum_{i=1}^{n} \alpha_{i}+\frac{2}{\hat{\sigma}^{2}} \sum_{i=1}^{n} \beta_{i} t_{i}
$$

\subsection{Modified maximum likelihood estimation under RSS}

Using the same approach in subsection (3.1), we can obtain the MMLEs under RSS. From (12) and (13) and under RSS, we can write the log-likelihood function as follows

$$
\ln L^{*}(\mu, \sigma)=-n \ln \sigma+\sum_{i=1}^{n} t_{i(i)}-\sum_{i=1}^{n}(n-i+1) e^{t_{i(i)}}+\sum_{i=1}^{n}(i-1) \ln \left(1-e^{t_{i(i)}}\right)
$$

where $t_{i(i)}=\left(z_{i(i)}-\mu\right) / \sigma$. To obtain the MMLEs, we expand the expression $g_{1}\left(t_{i(i)}\right)=$ $e^{t_{i(i)}}$ and $g_{2}\left(t_{i(i)}\right)=\frac{e^{t_{i(i)}-e^{t_{i(i)}}}}{1-e^{-e^{t_{i(i)}}}}$ in Taylor series around the points $v_{i}=\ln \left(-\ln \left(1-p_{i}\right)\right)$ and $p_{i}=i / n+1$. Using only the first two terms, We get

and

$$
g_{1}\left(t_{i(i)}\right)=\alpha_{1 i}+\beta_{1 i} t_{i(i)}
$$

where

$$
g_{2}\left(t_{i(i)}\right)=\alpha_{2 i}+\beta_{2 i} t_{i(i)}
$$

and

$$
\alpha_{1 i}=e^{v_{i}}\left(1-v_{i}\right), \quad \beta_{1 i}=e^{v_{i}}
$$

$$
\alpha_{2 i}=\frac{e^{v_{i}-e^{v_{i}}}}{1-e^{-e^{v_{i}}}}, \quad \beta_{2 i}=\frac{\left[e^{v_{i}-e^{v_{i}}}\left(1-e^{-e^{v_{i}}}\right)\left(1-e^{v_{i}}\right)\right]-e^{-2\left(v_{i}-e^{v_{i}}\right)}}{\left(1-e^{-e^{v_{i}}}\right)^{2}}
$$

Using these linear approximations, we can obtain the modified likelihood equations as

$$
-\frac{n}{\hat{\sigma}}+\frac{1}{\hat{\sigma}} \sum_{i=1}^{n}(n-i+1)\left(\alpha_{1 i}+\beta_{1 i} t_{i(i)}\right)-\frac{1}{\hat{\sigma}} \sum_{i=1}^{n}(i-1)\left(\alpha_{2 i}+\beta_{2 i} t_{i(i)}\right)=0
$$

and 


$$
\begin{array}{r}
-\frac{n}{\hat{\sigma}}-\frac{1}{\hat{\sigma}} \sum_{i=1}^{n} t_{i(i)}+\frac{1}{\hat{\sigma}} \sum_{i=1}^{n}(n-i+1) t_{i(i)}\left(\alpha_{1 i}+\beta_{1 i} t_{i(i)}\right) \\
-\frac{1}{\hat{\sigma}} \sum_{i=1}^{n}(i-1) t_{i(i)}\left(\alpha_{2 i}+\beta_{2 i} t_{i(i)}\right)=0
\end{array}
$$

The solutions of these equations are

and

$$
\hat{\mu}_{M M L-R S S}=A+\hat{\sigma} B
$$

$$
\hat{\sigma}_{M M L-R S S}=\frac{-D+\sqrt{D^{2}+4 n E}}{2 n}
$$

where

$$
\begin{gathered}
A=\frac{\left[\sum_{i=1}^{n}(i-1) \beta_{2 i} z_{i(i)}-\sum_{i=1}^{n}(n-i+1) \beta_{1 i} z_{i(i)}\right]}{\left[\sum_{i=1}^{n}(i-1) \beta_{2 i}-\sum_{i=1}^{n}(n-i+1) \beta_{1 i}\right]}, \\
B=\frac{\left[n-\sum_{i=1}^{n}(n-i+1) \alpha_{1 i}+\sum_{i=1}^{n}(i-1) \alpha_{2 i}\right]}{\left[\sum_{i=1}^{n}(i-1) \beta_{2 i}-\sum_{i=1}^{n}(n-i+1) \beta_{1 i}\right]}, \\
D=\sum_{i=1}^{n}\left[1-(n-i+1) \alpha_{1 i}+(i-1) \alpha_{2 i}\right]\left(z_{i(i)}-\hat{\mu}\right)
\end{gathered}
$$

and

$$
E=\sum_{i=1}^{n}\left[(n-i+1) \beta_{1 i}-(i-1) \beta_{2 i}\right]\left(z_{i(i)}-\hat{\mu}\right)^{2}
$$

The elements of the observed information matrix with respect to $\mu$ and $\sigma$ are as follows

$$
\begin{aligned}
& -\left.\frac{\partial^{2} \ln L^{*}}{\partial \mu^{2}}\right|_{\widehat{\mu}, \widehat{\sigma}} \cong \frac{1}{\hat{\sigma}^{2}} \sum_{i=1}^{n}(n-i+1) \beta_{1 i}-\frac{1}{\hat{\sigma}^{2}} \sum_{i=1}^{n}(i-1) \beta_{2 i}, \\
-\left.\frac{\partial^{2} \ln L^{*}}{\partial \sigma^{2}}\right|_{\widehat{\mu}, \hat{\sigma}} \cong & -\frac{n}{\hat{\sigma}^{2}}-\frac{2}{\hat{\sigma}^{2}} \sum_{i=1}^{n} t_{i(i)}+\frac{2}{\hat{\sigma}^{2}} \sum_{i=1}^{n}(n-i+1) \alpha_{1 i} t_{i(i)}+\frac{3}{\hat{\sigma}^{2}} \sum_{i=1}^{n}(n-i+1) \beta_{1 i} t_{i(i)}^{2} \\
& -\frac{2}{\hat{\sigma}^{2}} \sum_{i=1}^{n}(i-1) \alpha_{2 i} t_{i(i)}-\frac{3}{\hat{\sigma}^{2}} \sum_{i=1}^{n}(i-1) \beta_{2 i} t_{i(i)}^{2}
\end{aligned}
$$

and

$$
\begin{aligned}
-\left.\frac{\partial^{2} \ln L^{*}}{\partial \mu \partial \sigma}\right|_{\widehat{\mu}, \hat{\sigma}} \cong & -\frac{n}{\hat{\sigma}^{2}}+\frac{1}{\hat{\sigma}^{2}} \sum_{i=1}^{n}(n-i+1) \alpha_{1 i} \\
& +\frac{2}{\hat{\sigma}^{2}} \sum_{i=1}^{n}(n-i+1) \beta_{1 i} t_{i(i)}-\frac{1}{\hat{\sigma}^{2}} \sum_{i=1}^{n}(i-1) \alpha_{2 i} \\
& -\frac{2}{\hat{\sigma}^{2}} \sum_{i=1}^{n}(i-1) \beta_{2 i} t_{i(i)} .
\end{aligned}
$$

\section{Simulation study}


In this section, a simulation study is carried out using MATHCAD program to compare the performance of the ML and MML estimators based on SRS and RSS for IW distribution. The simulation study is conducted by choosing 1000 random samples of different samples size that were generated from IW distribution.

Using MATHCAD program, the ML and MML estimators based on SRS and RSS for IW distribution are obtained for different values of the parameters $(\lambda, \theta)=(0.5,2)$ and $(\lambda, \theta)=$ $(1.5,1.5)$ and different samples of sizes, $n=20,30,50,75$ and 100 under SRS and RSS, and using different values of $m$ and $k\{(m, k)=(4,5),(5,6),(5,10),(5,15)$ and $(10,10)\}$ in the case of RSS. The performance of the estimators of the parameters has been studied in terms of their MSEs, confidence length and PN probability. Pitman nearness probability can be calculated as

$$
P N=P\left\{\left|\hat{\theta}_{1}-\theta\right|<\left|\hat{\theta}_{2}-\theta\right|\right\}
$$

where $\hat{\theta}_{1}$ and $\hat{\theta}_{2}$ are the estimators of $\theta$. Based on PN probability criteria, we can say that $\hat{\theta}_{1}$ is better than $\hat{\theta}_{2}$ if $P N>0.5$ (Pitman (1937)). All of the calculation in this section were done using MATHCAD program version 2007 and based on 1000 replications. Proceedings of the simulation are described as follow:

Step 1: Suppose $U$ has a uniform $(0,1)$ distribution, then $x=(-\ln (u) / \lambda)^{-1 / \theta}$ follows the IW distribution. 1000 simple random samples of sizes 20,30,50,75 and 100 were generated from IW distribution.

Step 2: In the case of RSS, 1000 random samples of size $(m \times k)$ using different values of $m$ and $k\{(m, k)=(4,5),(5,6),(5,10),(5,15)$ and $(10,10)\}$ were generated from IW distribution. A random sample of size $(m \times k)$ is drawn by using RSS as in Figure (1), sampling was done without replacement from $\left(m^{2} \times k\right)$.

Step 3: Equations (4) and (5) were solved to obtain the ML estimates based on SRS and the MML estimates based on SRS are obtained by solving equations (14) and (15). Equations (10) were solved to obtain the ML estimates based on RSS and the MML estimates based on RSS are obtained by solving equations (17).

Step 4: The approximate confidence intervals of $\lambda$ and $\theta$ under SRS and SRS are obtained by the asymptotic variance covariance matrix using equations (8), equations (16), equations (11) and equations (18), respectively.

Step 5: The PN probability of the estimators $\hat{\lambda}_{M L E-S R S}, \hat{\theta}_{M L E-S R S}, \hat{\lambda}_{M L E-R S S}, \hat{\theta}_{M L E-R S S}$, $\hat{\lambda}_{M M L E-S R S}, \hat{\theta}_{M M L E-S R S}, \hat{\lambda}_{M M L E-R S S}$, and $\hat{\theta}_{M M L E-R S S}$ are calculated using equation (19).

The average value of estimates, MSE, confidence length and coverage probability under SRS and RSS are tabulated in Table 1 for $(\lambda, \theta)=(0.5,2)$ and in Table 4 for $(\lambda, \theta)=$ $(1.5,1.5)$. Tables 2 and 3 display the values of PN probability for $(\lambda, \theta)=(0.5,2)$, while Tables 5 and 6 present the PN probability for $(\lambda, \theta)=(1.5,1.5)$. From table 1 and 4 , it can be noted that in all the cases the MLEs and MMLEs of $\lambda$ and $\theta$ based on RSS have smaller MSEs than the corresponding estimates using SRS. It is also observed that the MMLEs of $\lambda$ and $\theta$ based on RSS perform better than the corresponding MLEs in terms of MSE and confidence length in most cases.

From Tables 2, 3, 5 and 6, we can see that the PN probability of the estimates based on RSS with respect to the estimates based on SRS are greater than 0.5, which show the outperform of the estimates based on RSS. Furthermore, the PN values show that the MMLEs under RSS perform better than MLEs under RSS and MLEs and MMLEs under SRS. 
Table 1. The average values of the ML estimates and MML estimates of $(\lambda, \theta)=(0.5,2)$, MSE in parentheses (first row of each cell), 95\% confidence lengths and the coverage probabilities in parentheses (second row of each cell), under SRS and RSS.

\begin{tabular}{|c|c|c|c|c|c|c|}
\hline$n$ & $(m ; k)$ & Parameter & \multicolumn{2}{|c|}{ ML Estimates } & \multicolumn{2}{|c|}{ MML Estimates } \\
\hline & & & SRS & RSS & SRS & RSS \\
\hline \multirow[t]{4}{*}{20} & \multirow[t]{4}{*}{$(4 ; 5)$} & \multirow[t]{2}{*}{$\lambda$} & $0.497(0.0249)$ & $0.492(0.0100)$ & $0.511(0.0263)$ & $0.513(0.0102)$ \\
\hline & & & $0.522(87.200)$ & $0.190(65.900)$ & $0.489(92.200)$ & $0.153(64.600)$ \\
\hline & & \multirow[t]{2}{*}{$\theta$} & $2.172(0.2151)$ & $2.183(0.1668)$ & $2.162(0.2108)$ & $2.102(0.1370)$ \\
\hline & & & $1.136(83.300)$ & $0.735(69.100)$ & $1.707(89.900)$ & $0.771(71.900)$ \\
\hline \multirow[t]{4}{*}{30} & \multirow[t]{4}{*}{$(5 ; 6)$} & \multirow[t]{2}{*}{$\lambda$} & $0.503(0.0165)$ & $0.491(0.0065)$ & $0.513(0.0172)$ & $0.506(0.0065)$ \\
\hline & & & $0.432(88.900)$ & $0.128(58.600)$ & $0.396(92.900)$ & $0.102(57.500)$ \\
\hline & & \multirow[t]{2}{*}{$\theta$} & $2.104(0.1119)$ & $2.125(0.0936)$ & $2.101(0.1103)$ & $2.065(0.0786)$ \\
\hline & & & $0.908(85.500)$ & $0.499(63.000)$ & $1.281(91.000)$ & $0.512(65.900)$ \\
\hline \multirow[t]{4}{*}{50} & \multirow[t]{4}{*}{$(5 ; 10)$} & \multirow[t]{2}{*}{$\lambda$} & $0.503(0.0094)$ & $0.494(0.0037)$ & $0.509(0.0097)$ & $0.503(0.0037)$ \\
\hline & & & $0.336(91.000)$ & $0.078(48.200)$ & $0.301(92.200)$ & $0.061(44.400)$ \\
\hline & & \multirow[t]{2}{*}{$\theta$} & $2.056(0.0542)$ & $2.085(0.0517)$ & $2.055(0.0538)$ & $2.047(0.0441)$ \\
\hline & & & $0.695(89.100)$ & $0.305(52.900)$ & $0.937(93.600)$ & $0.309(55.700)$ \\
\hline \multirow[t]{4}{*}{75} & \multirow[t]{4}{*}{$(5 ; 15)$} & \multirow[t]{2}{*}{$\lambda$} & $0.504(0.0062)$ & $0.496(0.0024)$ & $0.508(0.0064)$ & $0.502(0.0024)$ \\
\hline & & & $0.275(92.100)$ & $0.052(41.100)$ & $0.244(94.300)$ & $0.041(40.200)$ \\
\hline & & \multirow{2}{*}{$\theta$} & $2.030(0.0389)$ & $2.055(0.0318)$ & $2.030(0.0388)$ & $2.030(0.0284)$ \\
\hline & & & $0.562(85.800)$ & $0.205(46.300)$ & $0.741(93.100)$ & $0.206(45.900)$ \\
\hline \multirow[t]{4}{*}{100} & \multirow[t]{4}{*}{$(10 ; 10)$} & \multirow[t]{2}{*}{$\lambda$} & $0.501(0.0047)$ & $0.497(0.0013)$ & $0.504(0.0047)$ & $0.501(0.0013)$ \\
\hline & & & $0.238(90.600)$ & $0.039(42.200)$ & $0.210(93.900)$ & $0.031(39.100)$ \\
\hline & & \multirow{2}{*}{$\theta$} & $2.029(0.0299)$ & $2.039(0.0168)$ & $2.030(0.0300)$ & $2.020(0.0152)$ \\
\hline & & & $0.486(85.100)$ & $0.155(47.500)$ & $0.636(92.000)$ & $0.155(48.600)$ \\
\hline
\end{tabular}

Table 2. PN comparison between different estimators of $\lambda=0.5$

\begin{tabular}{|c|c|c|c|c|c|}
\hline \multirow{2}{*}{ PN probability of } & \multicolumn{5}{|c|}{ (cycle size; number of cycles) } \\
\hline & $(4 ; 5)$ & $(5 ; 6)$ & $(5 ; 10)$ & $(5 ; 15)$ & $(10 ; 10)$ \\
\hline$\hat{\lambda}_{M L E-R S S} v S \hat{\lambda}_{M L E-S R S}$ & 0.6410 & 0.6410 & 0.6380 & 0.6360 & 0.6820 \\
\hline$\hat{\lambda}_{M L E-R S S} v S \hat{\lambda}_{M M L E-R S S}$ & 0.4870 & 0.4670 & 0.4660 & 0.4740 & 0.4850 \\
\hline$\hat{\lambda}_{M L E-R S S} v S \hat{\lambda}_{M M L E-S R S}$ & 0.6440 & 0.6410 & 0.6410 & 0.6410 & 0.6800 \\
\hline$\hat{\lambda}_{M L E-S R S} v S \hat{\lambda}_{M M L E-R S S}$ & 0.3580 & 0.3680 & 0.3520 & 0.3610 & 0.3220 \\
\hline$\hat{\lambda}_{M L E-S R S} v S \hat{\lambda}_{M M L E-S R S}$ & 0.4960 & 0.5010 & 0.4920 & 0.5200 & 0.5010 \\
\hline$\hat{\lambda}_{M M L E-R S S} v S \hat{\lambda}_{M M L E-S R S}$ & 0.6550 & 0.6490 & 0.6440 & 0.6440 & 0.6830 \\
\hline
\end{tabular}

Table 3. PN comparison between different estimators of $\theta=2$

PN probability of (cycle size; number of cycles)

\begin{tabular}{|l|l|l|l|l|l|}
\hline & $(4 ; 5)$ & $(5 ; 6)$ & $(5 ; 10)$ & $(5 ; 15)$ & $(10 ; 10)$ \\
\hline
\end{tabular}




\begin{tabular}{|c|c|c|c|c|c|}
\hline$\hat{\theta}_{M L E-R S S} v S \hat{\theta}_{M L E-S R S}$ & 0.5430 & 0.5100 & 0.5240 & 0.5280 & 0.5780 \\
\hline$\hat{\theta}_{M L E-R S S} v s \hat{\theta}_{M M L E-R S S}$ & 0.3890 & 0.3860 & 0.4090 & 0.4400 & 0.4140 \\
\hline$\hat{\theta}_{M L E-R S S} v s \hat{\theta}_{M M L E-S R S}$ & 0.5310 & 0.5090 & 0.5230 & 0.5330 & 0.5830 \\
\hline$\hat{\theta}_{M L E-S R S} v S \hat{\theta}_{M M L E-R S S}$ & 0.4370 & 0.4700 & 0.4660 & 0.4680 & 0.4040 \\
\hline$\hat{\theta}_{M L E-S R S} v S \hat{\theta}_{M M L E-S R S}$ & 0.4730 & 0.4680 & 0.4530 & 0.4970 & 0.5090 \\
\hline$\hat{\theta}_{M M L E-R S S}$ vs $\hat{\theta}_{M M L E-S R S}$ & 0.5630 & 0.5380 & 0.5280 & 0.5390 & 0.5940 \\
\hline
\end{tabular}

Table 4. The average values of the ML estimates and MML estimates of $(\lambda, \theta)=(1.5,1.5)$, MSE in parentheses (first row of each cell), 95\% confidence lengths and the coverage probabilities in parentheses (second row of each cell), under SRS and RSS.

\begin{tabular}{|c|c|c|c|c|c|c|}
\hline$n$ & $(m ; k)$ & Parameter & \multicolumn{2}{|c|}{ ML Estimates } & \multicolumn{2}{|c|}{ MML Estimates } \\
\hline & & & SRS & RSS & SRS & RSS \\
\hline \multirow[t]{4}{*}{20} & \multirow[t]{4}{*}{$(4 ; 5)$} & $\lambda$ & $1.600(0.1780)$ & $1.625(0.1181)$ & $1.638(0.1930)$ & $1.621(0.1087)$ \\
\hline & & & $1.424(93.600)$ & $0.564(66.100)$ & $1.566(92.200)$ & $0.485(64.600)$ \\
\hline & & $\theta$ & $1.629(0.1210)$ & $1.637(0.0938)$ & $1.622(0.1186)$ & $1.576(0.0771)$ \\
\hline & & & $0.950(88.500)$ & $0.551(69.100)$ & $1.280(89.900)$ & $0.578(71.900)$ \\
\hline \multirow[t]{4}{*}{30} & \multirow[t]{4}{*}{$(5 ; 6)$} & $\lambda$ & $1.579(0.1177)$ & $1.572(0.0557)$ & $1.607(0.1268)$ & $1.567(0.0517)$ \\
\hline & & & $1.142(94.500)$ & $0.365(59.500)$ & $1.240(92.900)$ & $0.315(57.500)$ \\
\hline & & $\theta$ & $1.578(0.0629)$ & $1.594(0.0526)$ & $1.576(0.0621)$ & $1.549(0.0442)$ \\
\hline & & & $0.759(89.100)$ & $0.374(63.000)$ & $0.960(91.000)$ & $0.384(65.900)$ \\
\hline \multirow[t]{4}{*}{50} & \multirow[t]{4}{*}{$(5 ; 10)$} & $\lambda$ & $1.545(0.0587)$ & $1.550(0.0308)$ & $1.562(0.0618)$ & $1.545(0.0287)$ \\
\hline & & & $0.861(93.100)$ & $0.218(49.700)$ & $0.925(92.200)$ & $0.188(44.400)$ \\
\hline & & $\theta$ & $1.542(0.0305)$ & $1.564(0.0291)$ & $1.541(0.0303)$ & $1.535(0.0248)$ \\
\hline & & & $0.580(92.000)$ & $0.292(52.900)$ & $0.703(93.600)$ & $0.232(55.700)$ \\
\hline \multirow[t]{4}{*}{75} & \multirow[t]{4}{*}{$(5 ; 15)$} & $\lambda$ & $1.530(0.0363)$ & $1.534(0.0204)$ & $1.542(0.0377)$ & $1.531(0.0193)$ \\
\hline & & & $0.695(94.200)$ & $0.145(40.100)$ & $0.742(94.300)$ & $0.125(40.900)$ \\
\hline & & $\theta$ & $1.522(0.0219)$ & $1.542(0.0191)$ & $1.523(0.0218)$ & $1.522(0.0171)$ \\
\hline & & & $0.468(89.700)$ & $0.154(44.200)$ & $0.556(93.100)$ & $0.155(44.700)$ \\
\hline \multirow[t]{4}{*}{100} & \multirow[t]{4}{*}{$(10 ; 10)$} & $\lambda$ & $1.522(0.0266)$ & $1.522(0.0098)$ & $1.532(0.0276)$ & $1.519(0.0023)$ \\
\hline & & & $0.598(94.100)$ & $0.108(40.700)$ & $0.637(93.900)$ & $0.093(39.100)$ \\
\hline & & $\theta$ & $1.522(0.0168)$ & $1.529(0.0094)$ & $1.522(0.0169)$ & $1.515(0.0086)$ \\
\hline & & & $0.405(88.400)$ & $0.116(47.500)$ & $0.477(92.000)$ & $0.116(48.600)$ \\
\hline
\end{tabular}

Table 5. PN comparison between different estimators of $\lambda=1.5$

\begin{tabular}{|c|c|c|c|c|c|}
\hline & \multicolumn{5}{|c|}{ (cycle size; number of cycles) } \\
\hline & $(4 ; 5)$ & $(5 ; 6)$ & $(5 ; 10)$ & $(5 ; 15)$ & $(10 ; 10)$ \\
\hline$\hat{\lambda}_{M L E-R S S} v s \hat{\lambda}_{M L E-S R S}$ & 0.5760 & 0.6000 & 0.5860 & 0.5840 & 0.6670 \\
\hline$\hat{\lambda}_{M L E-R S S}$ vs $\hat{\lambda}_{M M L E-R S S}$ & 0.3560 & 0.3960 & 0.3780 & 0.3680 & 0.3930 \\
\hline$\hat{\lambda}_{M L E-R S S}$ vs $\hat{\lambda}_{M M L E-S R S}$ & 0.5790 & 0.6090 & 0.5980 & 0.5750 & 0.6550 \\
\hline
\end{tabular}




\begin{tabular}{|l|c|c|c|c|c|}
\hline$\hat{\lambda}_{M L E-S R S} v S \hat{\lambda}_{M M L E-R S S}$ & 0.4090 & 0.3930 & 0.4020 & 0.4070 & 0.3310 \\
\hline$\hat{\lambda}_{M L E-S R S} v S \hat{\lambda}_{M M L E-S R S}$ & 0.5750 & 0.5720 & 0.5590 & 0.5370 & 0.5240 \\
\hline$\hat{\lambda}_{M M L E-R S S} v S \hat{\lambda}_{M M L E-S R S}$ & 0.5900 & 0.6150 & 0.6050 & 0.5840 & 0.6570 \\
\hline
\end{tabular}

Table 6. PN comparison between different estimators of $\theta=1.5$

\begin{tabular}{|c|c|c|c|c|c|}
\hline PN probability of & \multicolumn{5}{|c|}{ (cycle size; number of cycles) } \\
\hline & $(4 ; 5)$ & $(5 ; 6)$ & $(5 ; 10)$ & $(5 ; 15)$ & $(10,10)$ \\
\hline$\hat{\theta}_{M L E-R S S} v s \hat{\theta}_{M L E-S R S}$ & 0.5430 & 0.5100 & 0.5240 & 0.5160 & 0.5780 \\
\hline$\hat{\theta}_{M L E-R S S} v s \hat{\theta}_{M M L E-R S S}$ & 0.3890 & 0.3860 & 0.4090 & 0.4310 & 0.4140 \\
\hline$\hat{\theta}_{M L E-R S S} v s \hat{\theta}_{M M L E-S R S}$ & 0.5310 & 0.5090 & 0.5230 & 0.5140 & 0.5830 \\
\hline$\hat{\theta}_{M L E-S R S} v s \hat{\theta}_{M M L E-R S S}$ & 0.4370 & 0.4700 & 0.4660 & 0.4680 & 0.4040 \\
\hline$\hat{\theta}_{M L E-S R S} v s \hat{\theta}_{M M L E-S R S}$ & 0.4740 & 0.4690 & 0.4530 & 0.4970 & 0.5090 \\
\hline$\hat{\theta}_{M M L E-R S S} v s \hat{\theta}_{M M L E-S R S}$ & 0.5630 & 0.5380 & 0.5280 & 0.5290 & 0.5940 \\
\hline
\end{tabular}

\section{Numerical example}

In this section, we use a data set of Dumonceaux and Antle (1973) to show the applicability of the proposed estimators. The data set represents the maximum flood levels of the Susquehenna River at Harrisburg, Pennsylvenia over 20 four-year periods (1890-1969). The original data set which consists of 20 observations are

\begin{tabular}{|l|l|l|l|l|l|l|l|l|l|}
\hline 0.654 & 0.613 & 0.315 & 0.449 & 0.297 & 0.402 & 0.379 & 0.423 & 0.379 & 0.324 \\
\hline 0.269 & 0.740 & 0.418 & 0.412 & 0.494 & 0.416 & 0.338 & 0.392 & 0.484 & 0.265 \\
\hline
\end{tabular}

Maswadah (2003) analyzed these data and showed that the IW distribution provide a good fit to the data. Also, Maswadah (2003) obtained the MLE of $\lambda$ and $\theta$ from the complete data set as $\hat{\lambda}=0.0119$ and $\hat{\theta}=4.3138$. Here, we select a random sample of size 15 by using SRS and RSS, sampling was done with replacement. In RSS method, five matrices $3 \times 3$ are drawn and then applying the technique presented in Figure 1. The generated SRS observations are

\begin{tabular}{|l|l|l|l|l|}
\hline 0.269 & 0.416 & 0.449 & 0.324 & 0.412 \\
\hline 0.315 & 0.315 & 0.412 & 0.379 & 0.265 \\
\hline 0.449 & 0.338 & 0.449 & 0.338 & 0.449 \\
\hline
\end{tabular}

while the generated RSS observations are 


\begin{tabular}{|l|l|l|l|l|}
\hline 0.265 & 0.269 & 0.269 & 0.324 & 0.338 \\
\hline 0.416 & 0.418 & 0.423 & 0.423 & 0.449 \\
\hline 0.494 & 0.613 & 0.613 & 0.740 & 0.740 \\
\hline
\end{tabular}

Table 7 displays the different estimates of the unknown parameters under SRS and RSS as well as the corresponding confidence intervals bounds. From table 7, it is seen that for parameter $\lambda$ the MML method under SRS perform better than other methods in terms of confidence interval length, while for parameter $\theta$ the ML method under RSS perform better than other methods in terms of confidence length (CL).

Table 7. The ML and MML estimates of $\lambda$ and $\theta$ and the corresponding 95\% confidence intervals under SRS and RSS for real data set.

\begin{tabular}{|c|c|c|c|c|c|c|c|c|c|}
\hline Sampling & Parameter & MLE & LB & UB & CL & MMLE & LB & UB & CL \\
\hline \multirow{2}{*}{ SRS } & $\lambda$ & 0.002 & - & 0.005 & 0.006 & 0.002 & 0.001 & 0.004 & 0.002 \\
& $\theta$ & 5.641 & 0.001 & 6.767 & 2.252 & 5.635 & 4.079 & 9.111 & 5.032 \\
& & & 4.515 & & & & & & \\
\hline \multirow{2}{*}{ RSS } & $\lambda$ & 0.035 & 0.010 & 0.060 & 0.050 & 0.036 & 0.030 & 0.044 & 0.014 \\
& $\theta$ & 3.351 & 2.676 & 4.026 & 1.350 & 3.338 & 2.782 & 4.170 & 1.388 \\
\hline
\end{tabular}

\section{Conclusion}

In this paper, we have considered the estimation problem of the unknown parameters of the IW distribution based on SRS and RSS. We used the ML and MML methods of estimation to estimate the unknown parameters. It is noted that the ML estimators cannot be obtained in closed forms, so, the MMLEs have been presented. We used a simulation study to compare the performance of the different estimators in terms of their MSEs, confidence length and PN probability. It is noted that the MMLEs based on RSS perform very well relative to their MSE, confidence length and PN probability. This indicates that the estimation based on RSS method is more efficient than estimation based on SRS. 


\section{References}

[1] Abu-Dayyeh, W., Assrhani, A. and Ibrahim, K. (2013). Estimation of the shape and parameters of pareto distribution using ranked set sampling, Statistical papers. 54, 207-225.

[2] Al-Saleh, M.F., Ali Al-Hadhrami, S. (2003a). Parametric estimation for the location parameter for symmetric distributions using moving extremes ranked set sampling with application to trees data. Environmetrics. 14(3), 651-664.

[3] Al-Saleh, M.F., Ali Al-Hadhrami, S. (2003b). Estimation of the mean of the exponential distribution using moving extremes ranked set sampling. Statistical papers. 44, 367-382.

[4] Balci, S., Akkaya, A.D. and Ulgen, B.E. (2013). Modified maximum likelihood estimators using ranked set sampling. Journal of computational and applied mathematics. 238, 171-179.

[5] Calabria, R. and Pulcini, G. (1989). Confidence limits for reliability and tolerance limits in the inverse Weibull distribution. Reliability engineering and system safety. 24, 77-85.

[6] Calabria, R. and Pulcini, G. (1990). On the maximum likelihood and least squares estimation in the inverse Weibull distribution. Statistica applicata. 2, 53-66.

[7] Calabria, R. and Pulcini, G. (1992). Bayes probability intervals in a load-strength model. Communication in statistics- Theory and methods. 21, 3393-3405.

[8] Calabria, R., Pulcini, G. (1994). Bayesian 2-sample prediction for the inverse Weibull distribution, Communication in statistics- Theory and methods. 23, 1811-1824.

[9] Chen, Z., Bai, Z. and Sinha, B. K. (2004). Ranked set sampling theory and applications. New York. Springer.

[10] Chen, W., Xie, M. and Wu, M. (2014). Modified maximum likelihood estimator of scale parameter using moving extremes ranked set sampling. Communications in statistics - simulation and computation. 45, 2232-2240.

[11] David, H. A. and Nagaraja, H. N. (2003). Order statistics. Third edition. John Wiley and Sons, New York.

[12] Dey S., Salehi M. and Ahmadi J. (2016). Rayleigh distribution revisited via ranked set sampling. Metron. 75, 69-85.

[13] Dumonceaux, R. and Antle, C. E. (1973). Discrimination between the lognormal and Weibull distribution. Technometrics. 15, 923-926.

[14] Erto, P. (1989). Genesis, properties and identification of the inverse Weibull lifetime model (in italian). Statistica applicate. 2, 117-128.

[15] Hassan, A. and Nassr, S. G. (2018). The inverse Weibull generator of distributions: properties and applications. Journal of Data Science. 16(3), 723-742.

[16] Helu, A., Abu-sallih, M. and Alkam, O. (2010). Bayes estimation of Weibull distribution parameters using ranked set sampling. Communication in statistics- Theory and methods. 39, 2533-2551. 
[17] Hussian, A. M. (2014). Bayesian and maximum likelihood estimation for Kumaraswamy distribution based on ranked set sampling, American journal of mathimatics and statistics. 4(1), $30-37$.

[18] Johnson, N.L., Kotz, S., and Balakrishnan, N. (1994). Continuous univariate distributions. Vol. I, $2^{\text {nd }}$ ed. John Wiley and Sons, New York.

[19] Keller, A. Z. and Kamath, A. R. R. (1982). Alternative reliability models for mechanical systems. Proceeding of the third international conference on reliability and maintainability. 411-415.

[20] Maswadah, M. (2003). Conditional confidence interval estimation for the inverse Weibull distribution based on censored generalized order statistics. Journal of statistical computation and simulation. 73 (12), 887-898.

[21] McIntyre, G. A. (1952). A method for unbiased selective sampling using ranked sets. Australian journal of agricultural research. 3, 385-390.

[22] Musleh, R. M. and Helu, A. (2014). Estimation of the inverse Weibull distribution based on progressively censored data: Comparative study. Reliability engineering and system safety. 131, 216-227.

[23] Nassar, M. and Abo-Kasem, O. (2017). Estimation of the inverse Weibull parameters under adaptive type-II progressive hybrid censoring scheme. Journal of computational and applied mathematics. 315, 228-239.

[24] Pitman, E. J G. (1937). The "closest" estimates of statistical parameters. Math. Proc. Camb. Philos. Soc. 33, 212-222.

[25] Sadek, A. and Alharbi, F. (2014). Weibull-Bayesian analysis based on ranked set sampling. International journal of advanced statistics and probability. 2 (2), 114-123.

[26] Singh, S. K., Singh, U. and Kumar, D. (2013). Bayesian estimation of parameters of inverse Weibull distribution, Journal of applied statistics. 40(7), 1597-1607.

[27] Takahasi, K., Wakimoto, K. (1968). On unbiased estimates of the population mean based on the sample stratified by means of ordering. Annals of the institute of statistical mathematics. 20, 131. 
